\title{
Fucoxanthin, the constituent of Laminaria japonica, triggers AMPK-mediated cytoprotection and autophagy in hepatocytes under oxidative stress
}

\author{
Eun Jeong Jang ${ }^{1 \dagger}$, Sang Chan Kim" ${ }^{1 \dagger}$, Ju-Hee Lee ${ }^{1,2}$, Jong Rok Lee ${ }^{1}$, II Kon Kim³ , Su Youn Baek ${ }^{1 *}$ \\ and Young Woo Kim ${ }^{1,3^{*}}$ (D)
}

\begin{abstract}
Background: Laminaria japonica has frequently been used as a food supplement and drug in traditional oriental medicine. Among the major active constituents responsible for the bioactivities of $L$. japonica, fucoxanthin (FX) has been considered as a potential antioxidant. This study was conducted to examine the effects of L. japonica extract (LE) or FX against oxidative stress on hepatocytes and to elucidate the overall their cellular mechanisms of the effects.

Methods: We constructed an in vitro model with the treatment of arachidonic acid (AA) + iron in HepG2 cells to stimulate the oxidative damage. The cells were pre-treated with LJE or FX for $1 \mathrm{~h}$, and incubated with AA + iron. The effect on oxidative damage and cellular mechanisms of LE or FX were assessed by cytological examination and several biochemical assays under conditions with or without kinase inhibitiors.

Results: LE or FX pretreatment effectively blocked the pathological changes caused by AA + iron treatment, such as cell death, altered expression of apoptosis-related proteins such as procaspase-3 and poly (ADP-ribose) polymerase, and mitochondria dysfunction. Moreover, FX induced AMPK activation and AMPK inhibitor, compound C, partially reduced the protective effect of FX on mitochondria dysfunction. Consistent with AMPK activation, FX increased the protein levels of autophagic markers (LC3II and beclin-1) and the number of acridine orange stained cells, and decreased the phosphorylation of mTOR and simultaneously increased the phosphorylation of ULK1. And the inhibition of autophagy by 3-methylanine or bafilomycin A1 partially inhibited the protective effect of FX on mitochondria dysfunction.
\end{abstract}

Conclusion: These findings suggest that FX have the function of being a hepatic protectant against oxidative damages through the AMPK pathway for the control of autophagy.

Keywords: Fucoxanthin, Oxidative stress, AMPK, Autophagy, AMPK/mTOR/ULK-1 pathway

\section{Background}

Laminaria japonica, one of the most well known brown seaweeds, is referred to as "Dashima" in Korean, "Kombu" in Japanese, and "Haidai" in Chinese. L. japonica is widely used as a food supplement, as well as a drug for treatment of various diseases [1]. L. japonica has abundant bioactive components, including polyphenols, pigments,

\footnotetext{
*Correspondence: rhodeus@dhu.ac.kr; ywkim@dhu.ac.kr

${ }^{\dagger}$ Equal contributors

${ }^{1}$ College of Oriental Medicine, Daegu Haany University, Gyeongsan, Gyeongsangbuk-do 38610, South Korea

Full list of author information is available at the end of the article
}

polysaccharides, minerals and amino acids [1]. Among bioactive compounds in $L$. japonica, fucoxanthin (FX), a marine carotenoid, has remarkable biological properties, including anti-cancer, obesity and inflammation [1-4]. FX has attracted much attention as a potential antioxidant given its unique chemical structure (Fig. 2a), which includes an allenic bond, epoxide group, and hydroxyl group [5]. Liu et al. (2011) reported that FX significantly recovered cell proliferation and increased the levels of glutathione and decreased intracellular reactive oxygen species (ROS) induced by ferric nitrilotriacetate [6]. In recently, Seo et al. (2016) showed that FX inhibited lipid 
accumulation and ROS formation by controlling adipogenic and lipogenic factors and ROS-regulating enzymes during differentiation in 3 T3-L1 adipocytes [7]. It is indicated that FX can effectively protect against hepatotoxicity by reducing intracellular ROS, associated with the antioxidant effects of FX.

ROS is a group of molecules including superoxide anion, hydroxyl radical and hydrogen peroxide, mainly produced in the mitochondria [8]. However, excess ROS can be involved in oxidative stress that destroys the structure of vital biomolecules, potentially leading to cellular dysfunction and remodeling [9]. Oxidative stress is known to activate the AMP-activated protein kinase (AMPK) signaling system in neuronal, heart, muscle, pancreatic and liver cells $[10,11]$. Interestingly, AMPK is known to be involved in ROS-induced autophagy that promotes cell survival in response to cellular stress such as malnutrition, hypoxia or ischemia [12]. Indeed, oxygen and nutrient deprivation induce the activation of AMPK leading to autophagy by inhibition of mTORC1 and phosphorylation of ULK1 [13, 14].

Autophagy is an important cell pathway for cell homeostasis and survival by removing damaged organelles and intracellular microbial pathogens [15]. Hepatocytes may be particularly dependent on the underlying autophagy for normal physiological function due to their high biosynthetic activity. In addition, autophagy plays a crucial role in the non-alcoholic and alcoholic liver diseases, drug-induced hepatic damage, viral hepatitis, fibrosis, liver cancer and hepatic ischemia reperfusion injury [15-17]. In liver ischemia reperfusion injuries, autophagy provides a prosurvival activity allowing the cell to cope with nutrient starvation and anoxia [16]. During hepatitis B or C infection, the level of autophagy is typically increased to promote viral growth [17]. In hepatocellular carcinoma, the level of autophagy is thought to be involved in both tumorigenesis and tumor suppression $[18,19]$.

In this regard, we tested whether L. japonica and FX alleviated hepatic oxidative stress in an in vitro model, HepG2 cells established by arachidonic acid (AA) + iron. Specifically, we explored the abilities of FX in regulation of autophagy and the underlying molecular mechanisms of their effects.

\section{Methods}

\section{Reagents}

AA and Compound C (C.C) were purchased from Calbiochem (San Diego, CA, USA). Anti-phospho-ACC, phospho-LKB1, procaspase-3, PARP, Bcl $\mathrm{XL}_{\mathrm{L}}$, LC3 I/II, beclin-1, AMPK, and phospho-AMPK antibodies were obtained from Cell Signaling Technology (Beverly, MA, USA). Bal-A1 was purchased from Santa Cruz Biotechnology (Santa Cruz, CA, USA). Horseradish peroxidase- conjugated goat anti-rabbit, rabbit anti-goat, and goat anti-mouse IgGs were obtained from Zymed Laboratories (San Francisco, CA, USA). FX, acrydine orange hemi zinc chloride salt, 3-methyladenine (3-MA), anti- $\beta$-actin antibody and other reagents were purchased from SigmaAldrich (St. Louis, MO, USA).

\section{Preparation of the L. japonica extract (LJE)}

L. japonica was purchased from Daewon pharmacy (Daegu, Korea), which is standardized with a standard herb of L. japonica in Korea Food and Drug Administrations. The L. japonica $(100 \mathrm{~g})$ were extracted as previously described $[20,21]$. The yield of lyophilized LJE was estimated to be $1.19 \%$ based on the dried weight.

\section{Cell culture}

HepG2 cells, a human hepatocyte-derived cell line, were provided by American Type Culture Collection (Rockville, MD, USA), and cultured as previously described [20]. To simulate oxidative stress, cells were incubated with $10 \mu \mathrm{M}$ AA for $12 \mathrm{~h}$, followed by exposure to $5 \mu \mathrm{M}$ iron for $1 \mathrm{~h}$. The cells were treated with FX or LJE for $1 \mathrm{~h}$ prior to the incubation with AA at the indicated doses.

\section{Cell viability assay}

The cells were plated at a density of $1 \times 10^{5}$ cells per well in a 48-well for $24 \mathrm{~h}$ as previously described [20]. The media was incubated with $0.25 \mathrm{mg} / \mathrm{ml}$ MTT for $2 \mathrm{~h}$, and formazan crystals were dissolved with the addition of $200 \mu \mathrm{l}$ DMSO.

\section{Terminal deoxynucleotidyl transferase dUTP nick end labeling (TUNEL) assay}

The TUNEL assay was performed using the DeadEnd ${ }^{\mathrm{m}}$ Colorimetric TUNEL System, according to the manufacturer's instruction. The samples were washed and examined under light microscope.

\section{Western blot analysis}

The cells were plated at a density of $5 \times 10^{5}$ cells per well in a 6-well plate for $24 \mathrm{~h}$. After the treatment designated, cells were lysed in RIPA buffer (Thermo Scientific, Rockford, IL, USA) as previously described [20, 21]. The protein bands were detected using Fusion Solo scanning system (Vilber Lourmat, Paris, France), and quantified using Image J ver 1.42 software (NIH, Bethesda, USA).

\section{Measurement of ROS production}

DCFH-DA, a cell-permeable non-fluorescent probe, has been used as a substrate for quantitation of intracellular oxidant production in HepG2 cells [21]. After treatment of reagents, cells were stained with $10 \mu \mathrm{M}$ DCFH-DA for $30 \mathrm{~min}$ at $37{ }^{\circ} \mathrm{C}$. The fluorescence intensity in the cells was measured at an excitation/emission wavelength 
of $485 / 535 \mathrm{~nm}$, using in the cells measured in a microplate reader.

\section{Determinant of glutathione (GSH) content}

Intracellular GSH content was quantified using a commercial GSH BIOXYTECH GSH-400 kit (Oxis International, Portland, OR) according to the manufacturer's protocol, and the absorbance level was measured at $405 \mathrm{~nm}$.

\section{Flow cytometric analysis of $\Delta \Psi_{\mathrm{m}}$}

$\Delta \Psi \mathrm{m}$ was measured using rhodamine123 (Rh123). Following treatment, cells were stained with $0.05 \mu \mathrm{g} / \mathrm{ml}$ of Rh123 for $1 \mathrm{~h}$ and then harvested by trypsinization. The change in $\triangle \Psi \mathrm{m}$ was monitored using a FACS flow cytometer (Partec, Münster, Germany). In each analysis, a total of 10,000 events were recorded as previous described [20].

\section{Acridine orange $(\mathrm{AO})$ staining}

HepG2 cells were plated on 18-mm cover glasses and incubated for $24 \mathrm{~h}$ to reach at approximately $70 \%$ confluence. They were then incubated either in the presence or absence of $30 \mu \mathrm{M}$ FX, washed twice with PBS and fixed with ice-cold $4 \%$ paraformaldehyde for $10 \mathrm{~min}$ at room temperature. Subsequently, the cells were stained with $\mathrm{AO}(1 \mu \mathrm{g} / \mathrm{ml})$ for $15 \mathrm{~min}$ at room temperature, washed, and examined under a fluorescence microscope (Nikon, Japan).

\section{Profiling the content of fucoxanthin by ultra performance} liquid chromatography (UPLC)

Water ACQUITYTM ultraperformance LC system (USA) was used to assess UPLC analysis as previously described [20]. Waters ACQUITYTM BEH C18 column $(1.7 \mu \mathrm{m}, 2.1 \mathrm{~mm} \times 100 \mathrm{~mm})$ was used as Waters ACQUITYTM PDA and HPLC Column, and the fucoxanthin was analyzed at $330 \mathrm{~nm}$. The standard fucoxanthin was melted by methanol and diluted to make solution containing $1 \mu \mathrm{g} / \mathrm{ml}$. L. japonica $1 \mathrm{~g}$ was also added with methanol $10 \mathrm{ml}$, and then sonication was perfomed for $3 \mathrm{~h}$. After then, it was filtered through a $0.2 \mu \mathrm{m}$ filter (Nalgene, NY, USA). A mobile phase was a mixed liquid of the acetonitrile and water, and the analysis condition was as in Table 1 . The sample was injected with $2 \mu \mathrm{l}$, and a flow rate was $0.4 \mathrm{ml} / \mathrm{min}$.

\section{Statistical analysis for study}

GraphPad Prism software version 5.01 (Graph Pad Software, La Jolla, CA) was used for all statistical analyses as previously described [20, 21]. Significance levels were calculated by repeated measures of ANOVA with the Dunnett post hoc test under 95\% confidence interval. Data were presented as the mean with standard deviation (mean \pm S.D.). Within
Table 1 Solvent gradient for the analysis fucoxanthin in L. japonica

\begin{tabular}{llll}
\hline Time (min) & $\begin{array}{l}0.1 \% \mathrm{FA} / \\
\text { Water (\%) }\end{array}$ & $\begin{array}{l}0.1 \% \mathrm{FA} / \\
\text { Acetonitrile (\%) }\end{array}$ & $\begin{array}{l}\text { Flow rate } \\
(\mathrm{ml} / \mathrm{min})\end{array}$ \\
\hline 0 & 98 & 2 & 0.40 \\
1 & 98 & 2 & 0.40 \\
2 & 90 & 10 & 0.40 \\
4 & 70 & 30 & 0.40 \\
7 & 50 & 50 & 0.40 \\
9 & 30 & 70 & 0.40 \\
10 & 10 & 90 & 0.40 \\
12 & 0 & 100 & 0.40 \\
14 & 98 & 2 & 0.40 \\
16 & 98 & 2 & 0.40 \\
\hline
\end{tabular}

figures, the $P$ values were displayed with asterisks (*** $P<0.001, * * P<0.01, * P<0.05)$.

\section{Results}

L. japonica Extract (LJE) decreases AA + iron induced cytotoxicity in HepG2 cells

An MTT assay for cell viability indicated that LJE pretreatment $(3,10,30$, and $50 \mu \mathrm{g} / \mathrm{ml})$ significantly protected cells from the potential injury induced by AA + iron. Since the maximum cell viability was achieved at $30 \mu \mathrm{g} / \mathrm{ml}$ of LJE, the same concentration was applied in subsequent experiments (Fig. 1a). In western blot analysis, treatment of $\mathrm{AA}+$ iron markedly induced decreases in the protein levels of procaspase- 3 and $\mathrm{Bcl}_{\mathrm{XL}}$, verifying $\mathrm{AA}+$ iron induction of apoptosis, which was completely blocked by LJE pretreatment (Fig. 1b). Morphological examination by light microscopy and TUNEL assay (Fig. 1c) confirmed the cytoprotective effect of LJE against the synergized toxicity of AA + iron. After treatment with LJE, positive staining located in the nucleus by $\mathrm{AA}+$ iron were apparently decreased (Fig. 1c). To further examine the antioxidative effects of LJE, we measured the contents of GSH and ROS. The intracellular concentration of GSH was substantially decreased by AA + iron, but was recovered by LJE treatment. In contrast, treatment with LJE alone had no effects on cellular GSH levels (Fig. 1d). The ROS generation assay using DCFH-DA indicated that LJE treatment effectively abrogated increases in ROS production caused by AA + iron (Fig. 1e). The effect of LJE on $\mathrm{AA}+$ iron-induced loss of mitochondrial membrane potential $(\Delta \Psi \mathrm{m})$ monitored by FACS analysis of Rho123 staining (Fig. 1f). Rho123 fluorescence intensity was not significantly altered in LJE-treated cells compared to untreated controls, though AA + iron markedly reduced rhodamine fluorescence, indicating the loss of $\Delta \Psi \mathrm{m}$ (Fig. 1f). LJE treatment significantly restrored the loss of $\Delta \Psi \mathrm{m}$ caused by AA + iron (Fig. 1f). 
a

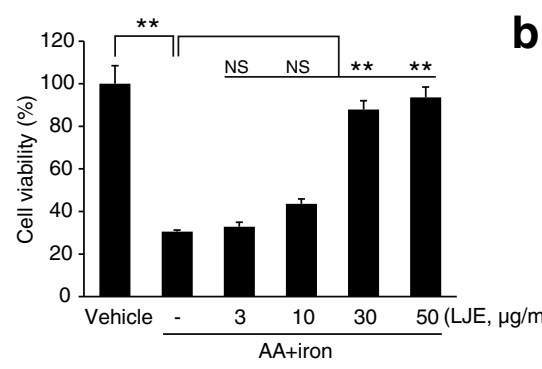

C
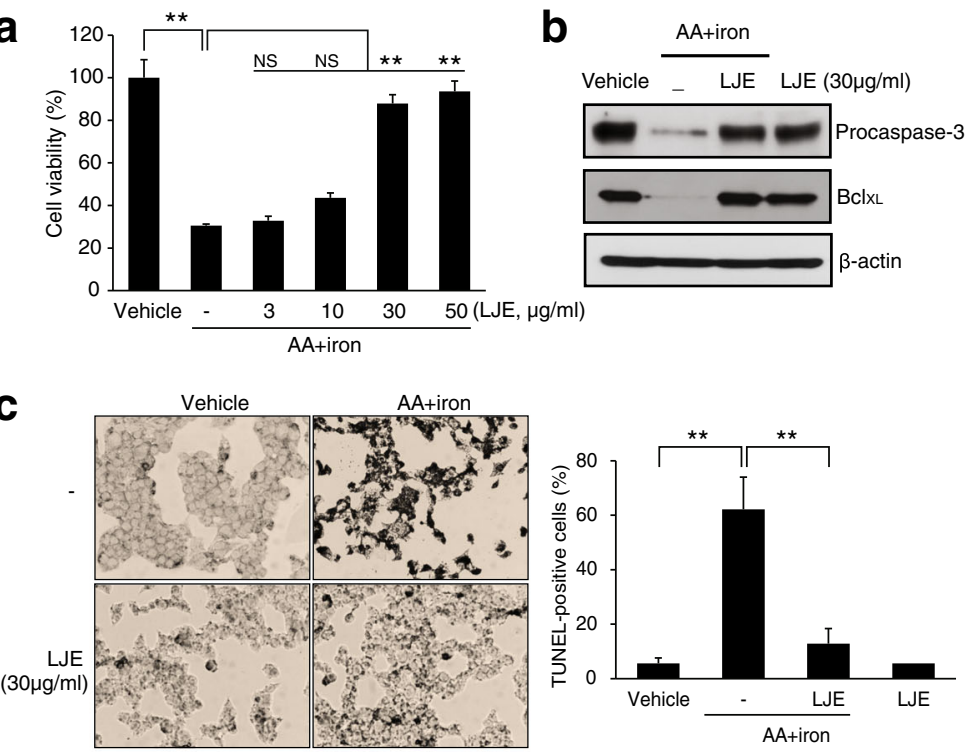
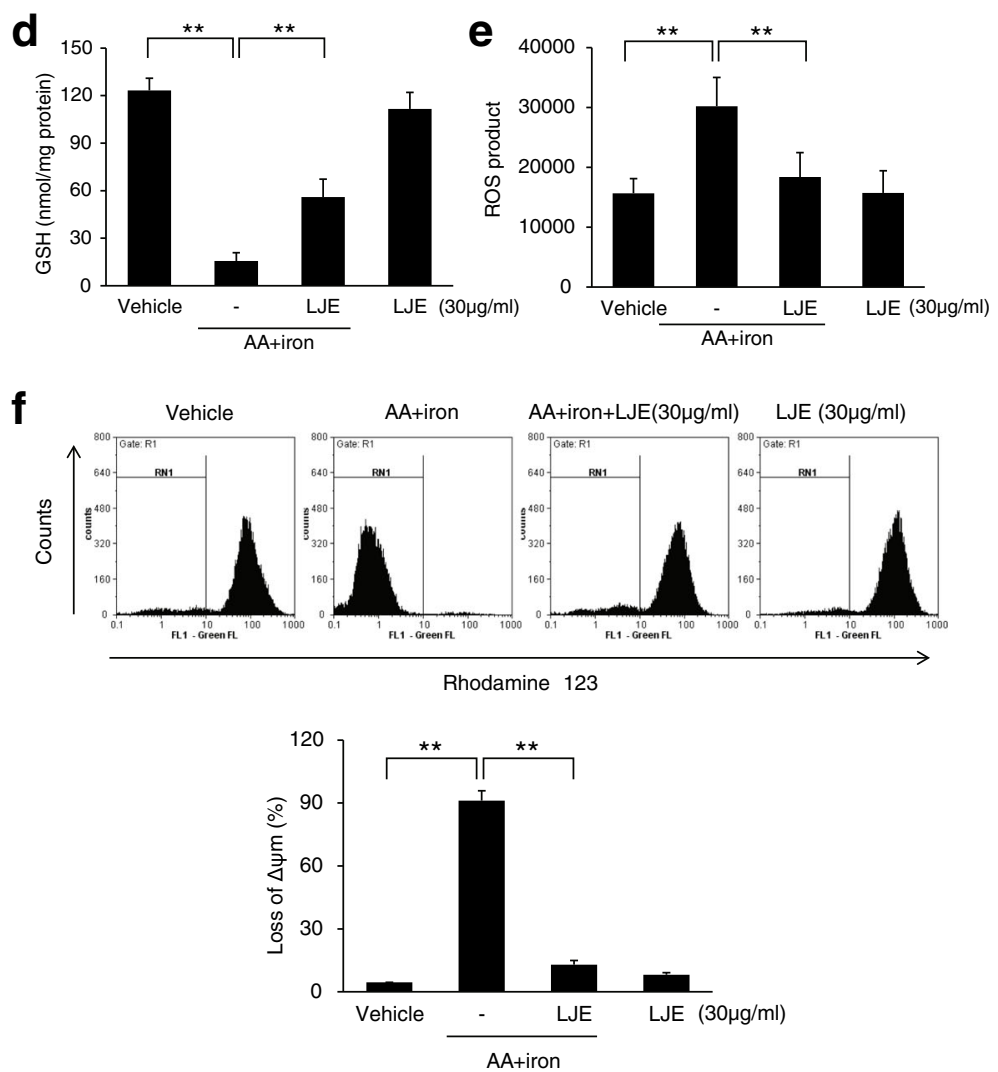

Fig. 1 L. japonica extract (LJE) decreases AA + iron induced cytotoxicity in HepG2 cells. HepG2 cells were incubated with indicated dose of LJE for $1 \mathrm{~h}$ and later treated with $10 \mu \mathrm{M}$ AA for $12 \mathrm{~h}$, being followed by exposure to $5 \mu \mathrm{M}$ iron for $3 \mathrm{~h}$. (a) Cell viability was assessed by the MTT assay. (b) Expression of proteins associated with apoptosis was determined by western blot analysis. Equal protein loading was verified by $\beta$-actin. (c) The levels of apoptosis in each groups examined by TUNEL assay. Representative images show apoptosis of HepG2 cells in vehicle control, LJE treated, AA + iron treated, and AA + iron treated with LJE groups (left). Percentage of TUNEL+ cell nuclei calculated relative to total number of cell nuclei (right). (d) Cellular GSH content was assessed in cells by using GSH assay kit. (e) Cellular reactive oxygen species production was monitored by measuring intensity of dichloro fluoresce in fluorescence. (f) $\Delta \psi \mathrm{m}$ depolarization monitored by FACS analysis of Rh123 staining. Relative proportions of low Rh-123 intensity (RN1 fractions) are expressed as the mean \pm S.D. of three separated experiments. For panel from A to E, data represent the mean \pm S.D. for the four replicates. ${ }^{* *} p<0.01$ 


\section{Fucoxanthin (FX) ameliorates AA + iron-induced cytotoxicity}

Next, we determined the effects of FX against oxidative stress induced by AA + iron (Fig. 2a). FX treatment inhibited death of cell induced by AA + iron, and this decrease in cell viability was recovered by pre-treatment with $30 \mu \mathrm{M}$ of FX (Fig. 2b). In western blot analysis, cleavage of PARP and caspase- 3 were strongly observed in AA + iron-treated cells, which were blocked by FX pretreatment (Fig. 2c). FX treatment significantly inhibited the change in $\Delta \Psi \mathrm{m}$ caused by AA + iron (Fig. 2d). These results indicate that FX remarkably suppressed AA + iron-induced collapse of $\Delta \Psi \mathrm{m}$, consequently protecting liver cells.
FX-induced the activation of AMPK alleviates cell damage by oxidative stress

Stimulation of FX $(30 \mu \mathrm{M})$ markedly induced the phosphorylation of AMPK (Fig. 3a). This compound also induced the phosphorylation of LKB1, an upstream kinase of AMPK, and ACC, a primary downstream target of AMPK (Fig. 3a). To determine the role of AMPK in protection of HepG2 cells by FX, we measured $\Delta \Psi \mathrm{m}$ levels after treating with a chemical inhibitor of AMPK, C.C. C.C inhibited the protective effect of FX on AA + ironinduced the loss of $\Delta \Psi \mathrm{m}$ in HepG2 cells (Fig. 3b). Collectively, these results suggest that FX activates the LKB1-AMPK signaling pathway, and that this activation

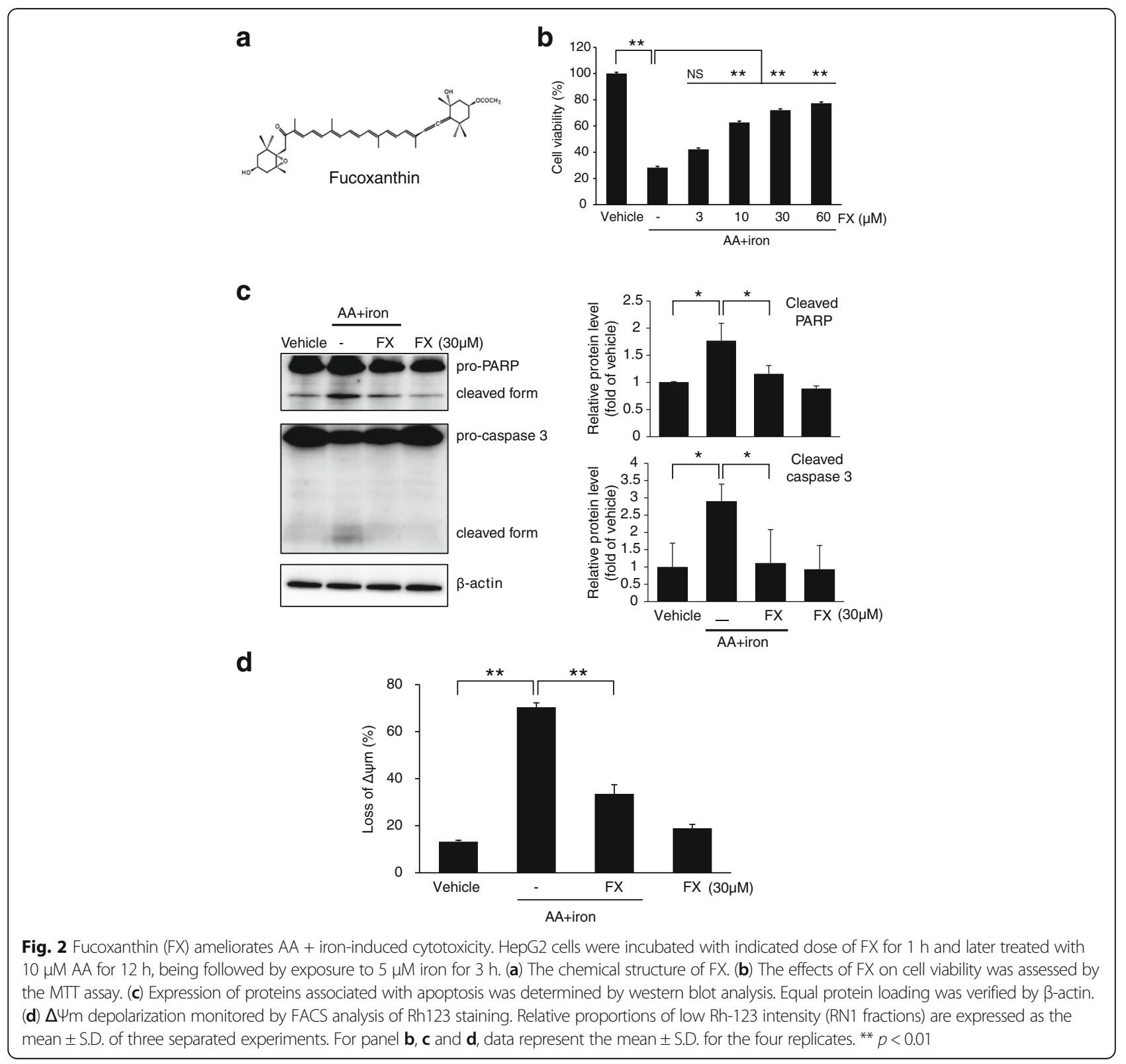



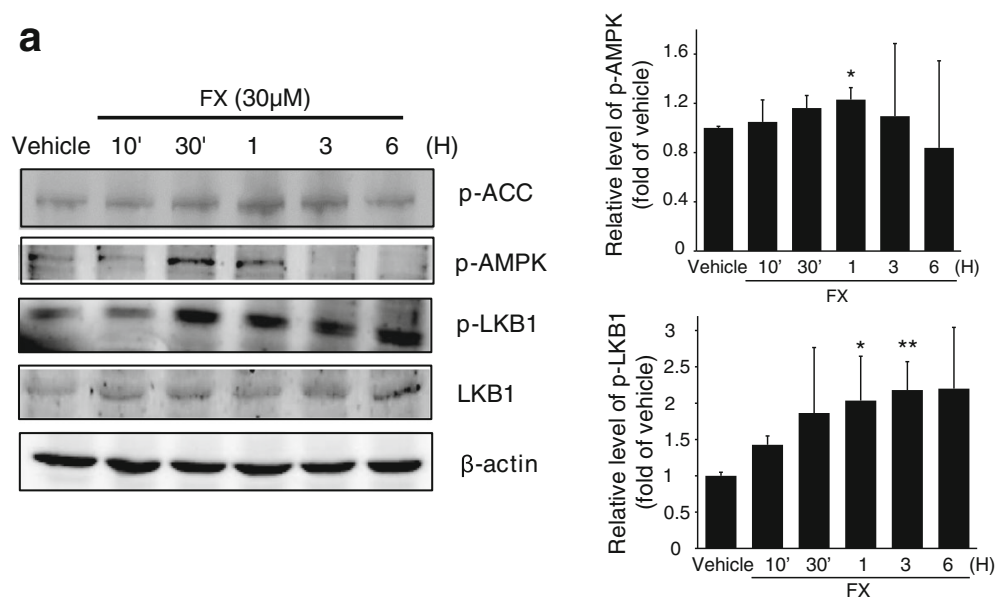

b

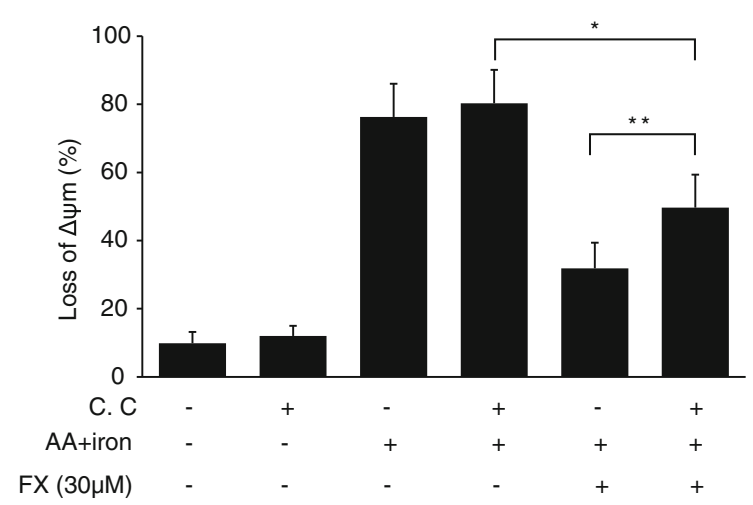

Fig. 3 FX-induced the activation of AMPK alleviates cell damage by oxidative stress. (a) FX induces phosphorylation of the proteins associated with AMPK pathway, ACC, LKB1 and AMPK. Western blot analyses were performed with the lysates of cells that had been treated with $30 \mu \mathrm{M}$ FX for the indicated time period. $\beta$-actin served as a loading control. Protein levels were presented as relative band intensities to control (vehicle treated) group. Results represent the mean \pm S.D. for three separate experiments. ${ }^{*} p<0.05 ;{ }^{* *} p<0.01$; ${ }^{* *} p<0.001$. (b) The effect of FX to restore $\Delta \psi \mathrm{m}$ was revered by C.C. Following treatment with $10 \mu \mathrm{M}$ C.C for $1 \mathrm{~h}$, cells were incubated with FX and/or AA + iron, and $\Delta \psi \mathrm{m}$ was evaluated with Rh123 stain by FACS. Data represent the mean \pm S.D. for four replicates. * $p<0.05$; ${ }^{* *} p<0.01$

of beneficial molecules is responsible for FX's inhibition of mitochondria damage induced by oxidative stress.

\section{FX triggers AMPK-dependent cytoprotective autophagy}

Treatment with $30 \mu \mathrm{M}$ FX upregulated beclin-1 and promoted the conversion of LC3 I to LC3 II as compared to the control (Fig. 4a). The AVOs were clearly observed in the HepG2 cells (red fluorescence) following treatment with FX (Fig. 4b). As shown in Fig. 4c, inhibition of AMPK activity by C.C markedly attenuated FXinduced accumulation of beclin-1, suggesting that AMPK is critical in the regulation of FX-induced autophagy. In addition, western blotting revealed that FX rapidly downregulated the phosphorylation of mTORC1 (Ser2448), which is known to negatively modulate autophagy and be inhibited by AMPK (Fig. 5a). In contrast, the phosphorylation levels of ULK1 (Ser555) was increased during FX treatment in time-dependent manner (Fig. 5a). Furthermore, we manipulated autophagy activity using 3-MA and Baf-A1 to suppress autophagy.
FACS analysis of $\triangle \Psi \mathrm{m}$ showed that 3-MA and Baf-A1 partially blocked the effect of FX on mitochondrial protection (Fig. 5b).

\section{Discussion}

In our results, LJE or FX ameliorated oxidative damage induced by $\mathrm{AA}+$ iron on hepatocytes, as confirmed by the inhibition of cell death and the restorating the loss of $\Delta \Psi \mathrm{m}$. We also demonstrated that these hepatoprotective effects of FX can be attributed to the function of autophagy via the AMPK/mTORC1/ULK-1 axis.

To determine the capacity of LJE or FX to reduce oxidative damage, we employed an in vitro model, HepG2 cells treated with AA + iron. In liver, the level of iron is tightly regulated by the control of absorption, storage and recycling, which is critical for the protection of liver tissues as well as other organ tissues from iron-induced cellular damages [21]. However, a chronic increase of iron level in liver can result in excess ROS production and liver injury, such as steatohepatitis, fibrosis, 


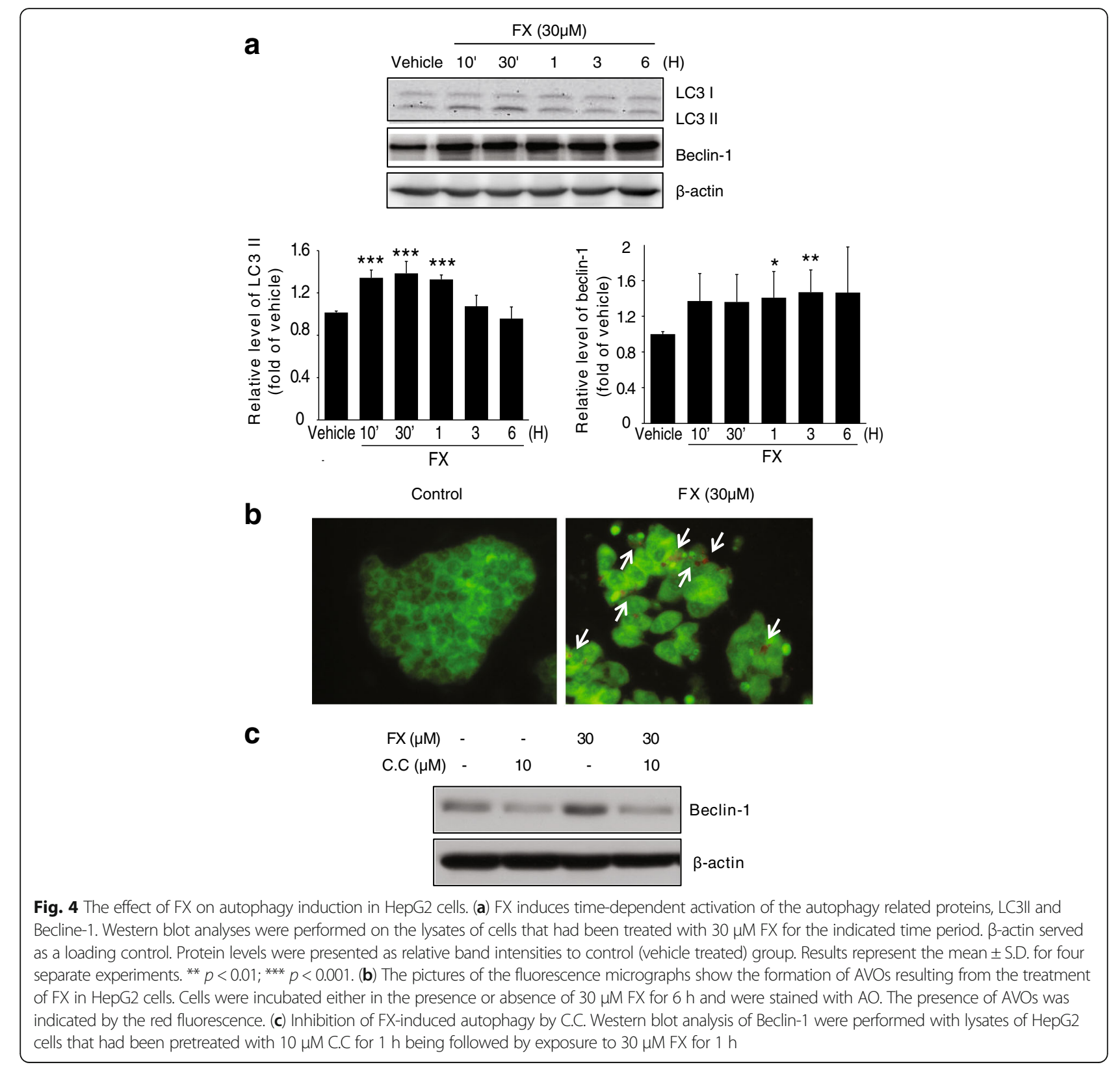

cirrhosis, and hepatocellular carcinoma [22]. The release of AA can also be induced by an increase in oxidative stress that originates from excessive levels of iron [23]. Although prostaglandins, essential components in cellular protection, are produced from AA, excessive AA can induce extremely high levels of cellular and mitochondrial ROS, negatively influencing the functions of several processes related to mitochondrial respiration [23]. The combinatorial treatment of AA and iron can thus reduce cell viability, and this treatment may be used to test the potential of cytoprotective agents targeting mitochondria against severe oxidative stress. In this study, AA + iron successfully induced cell death, production of ROS and damage of mitochondria in HepG2 cells. However, pretreatment of LJE or FX significantly blocked the ability of AA + iron to induce similar detrimental effects in HepG2 cells.

Recent studies have shown that AMPK serves as a key regulator of hepatocytes viability under oxidative stress $[10,11,23]$. Indeed, many natural compounds, such as resveratrol, sauchinone, and isoliquiritigenin, have been reported to protect hepatocytes by inhibiting production of ROS and mitochondrial dysfunction through activation of AMPK [24-26]. In our study, FX upregulated the phosphorylation of AMPK, ACC (the downstream target) and LKB1 (the essential upstream kinase) in hepatocytes. Furthermore, the inhibition of AMPK using compound $\mathrm{C}$ reduced the beneficial effect of FX on 

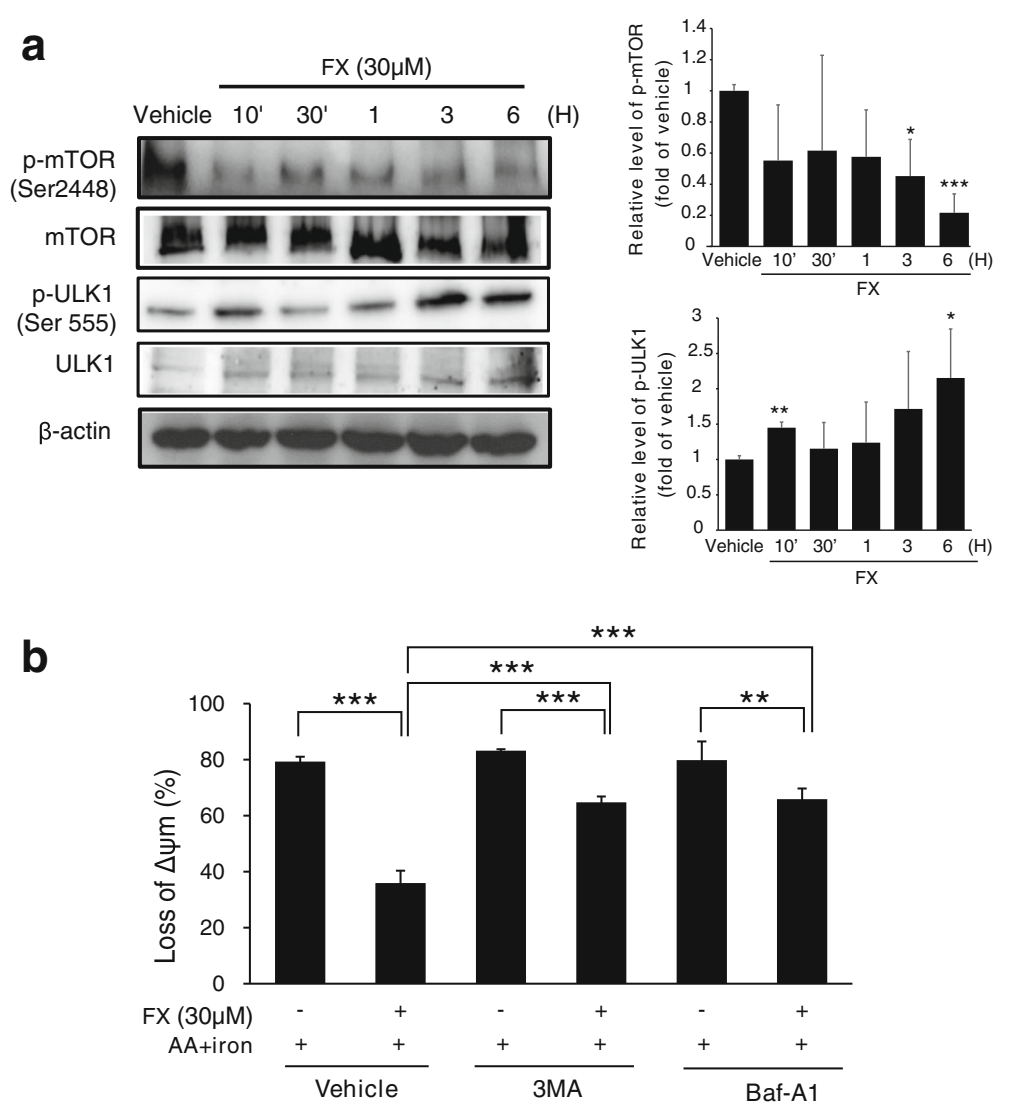

Fig. 5 The role of AMPK activation by FX in autophagy induction. (a) Western blot analysis for the phosphorylated level of mTOR (Ser2448) and ULK1 (Ser555) in HepG2 cells treated with $30 \mu \mathrm{M}$ FX for indicated time period. Results represent the mean \pm S.D. for four separate experiments. ${ }^{*} p<0.05$; ${ }^{* *} p<0.01$; *** $p<0.001$. (b) The effect of FX to restore $\Delta \psi \mathrm{m}$ was revered by 3-MA and Baf-A1. After 3-MA and Baf-A1 treatment (5 $\mu \mathrm{M}$ for $1 \mathrm{~h}$, respectively), cells were incubated with FX for $1 \mathrm{~h}$, being followed by the addition of AA (for $12 \mathrm{~h})+$ iron ( $1 \mathrm{~h}$ ). Data represent the mean \pm S.D. for three replicates. ${ }^{* *} p<0.01 ;{ }^{* * *} p<0.001$

mitochondria. Those results partially suggest that FX protects cells through activation of AMPK.

It is now widely accepted that AMPK induce autophagy, in turn, serving to reduce oxidative damage [12-14]. Autophagy can suppress cell death by eliminating damaged organelles or unnecessary cellular components formed from a variety of stresses, thereby playing adaptive roles to protect organisms against infections, cancer, neurodegeneration, aging, and heart diseases [15-18, 27]. The process of autophagy involves formation of double membrane vesicles (autophagosome) that enwrap portions of the cytoplasm [24]. To detect the development of AVOs, HepG2 cells treated with FX were stained with acridine orange. Our results demonstrated that the bright red fluorescence significantly increased after FX treatment, indicating the development of AVOs. Western blotting studies also demonstrated that FX induced HepG2 cell autophagy, as shown by the conversion of LC3B-I in LC3BII and the expression of Beclin-1, indicators of autophagy $[24,28]$. Furthermore, AMPK inhibition by a chemical inhibitor of AMPK $\alpha$, C.C abolished the increased protein level of beclin-1 by FX. Consequently, our current findings suggest that the activation of AMPK by FX be involved in induction of autophagy in HepG2 cells.

AMPK activation promoted autophagy through direct activation of ULK1 and inhibition of mTORC1, a negative regulator of autophagy [14]. mTORC1 is important in the autophagy and its activity inhibits autophagy by ULK1 phosphorylation, which induce disassociation between ULK1 and AMPK [14]. In this study, we observed a significant increase in the levels of ULK1 and AMPK phosphorylation in response to FX. Thus, our findings indicate a model in which FX induces autophagy by activating the AMPK-ULK1-mTORC1 axis. Finally, present study showed that 3-MA and Baf-A1, the autophagy inhibitors, partially reversed the inhibitory effect of FX on AA + iron-induced mitochondrial membrane potential depolarization in HepG2 cells, suggesting the involvement of AMPK-induced autophagy as the hepatoprotective activity of FX in HepG2 cells.

Although there are various component in L. japonica, in this study, we confirmed the content of FX (mass 


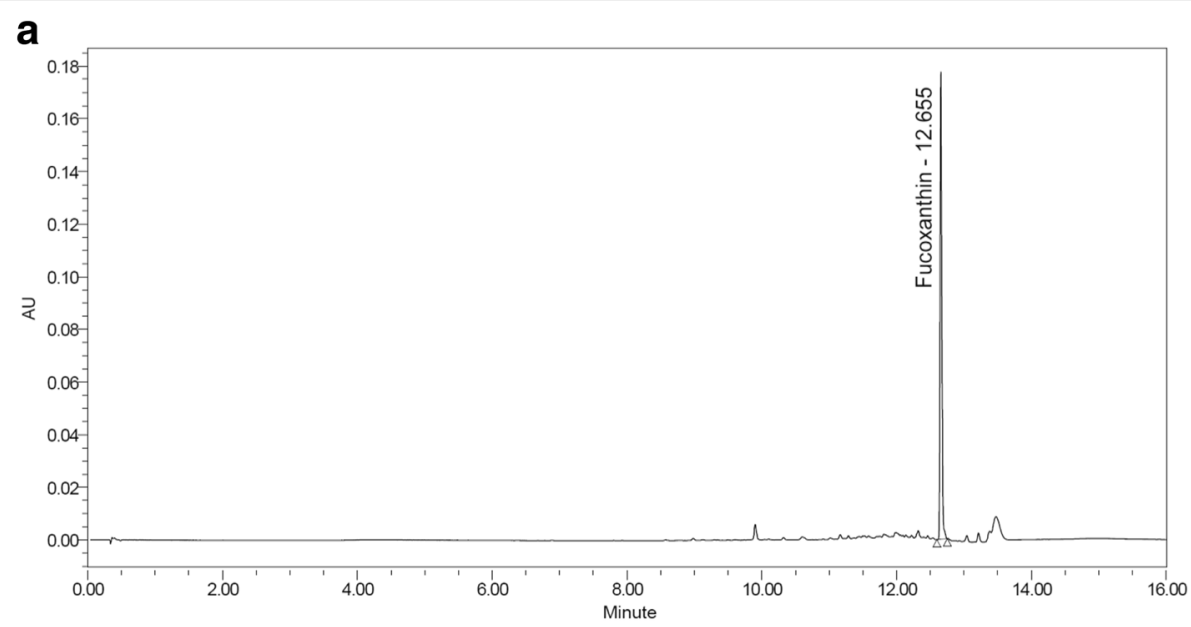

b

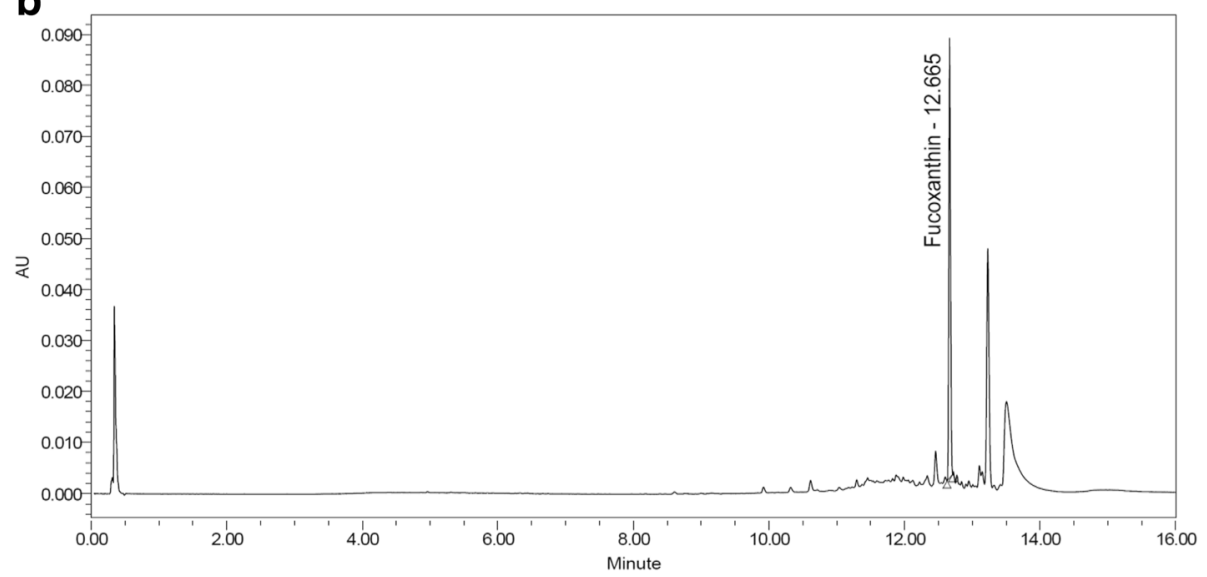

Fig. 6 UPLC chromatogram of fucoxanthin standard (a) and fucoxanthin in L. japonica (b). The peak represents fucoxanthin (330 nm)

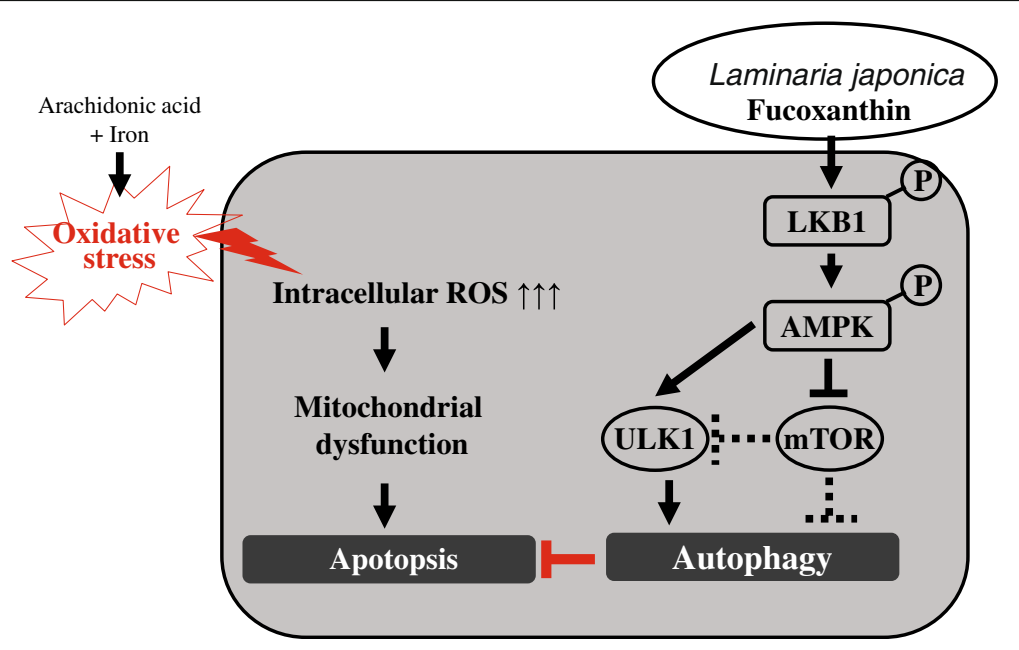

Fig. 7 Schematic diagram showed that FX induces AMPK-mediated autophagy contributing to ameliorates oxidative stress in HepG2 cells 
accuracy; 28.197 ppm) in L. japonica (Fig. 6). Recently, some report also showed that FX is known to significantly inhibit on the proliferation of HepG2 cells [29]. The result showed that both the protein degradation and transcriptional repression was responsible for cyclin $\mathrm{D}$ suppression by FX in HepG2 cells by inducing G1 arrest as mediated with GADD45A and MAPK pathway [29]. But, in the present study, FX showed a significantly protective effect on HepG2 cells against AA + iron-induced oxidative injury. Therefore, the active compound in the L. japonica and FX in the aspect of cell protection as well as their machanism remains to be further established.

\section{Conclusion}

Our results suggested that FX could protect hepatocytes against AA + iron-induced oxidative stress and trigger autophagy, which is likely associated with the LKB1AMPK $\alpha$ signaling pathway (Fig. 7). The current study also showed that FX or Laminaria japonica likely contributed to further understanding of its potential use as a hepatic protectant and nutraceutical.

\begin{abstract}
Abbreviations
3-MA: 3-methyladenine; AA: Arachidonic acid; ACC: Acetyl-CoA carboxylase; AMPK: AMP-activated protein kinase; ATG: Autophagy-related proteins; AVO: Acidic vesicular organelle; Bal-A1: Bafilomycin A1; BCl-xL: B-cell lymphoma-extra large; C.C: Compound C; DCFH-DA: 2',7'-Dichlorofluorescein diacetate; DMSO: Dimethyl sulphoxide; FBS: Fetal bovine serum; FX: Fucoxanthin; GSH: Glutathione: LC3: Microtubule-associated protein 1 light chain 3; LE: L. japonica extract; LKB1: Liver kinase B1; mTOR: mammalian target of rapamycin; MTT: 3-(4,5dimethylthiazol-2-yl)-2,5-diphenyl-tetrazolium bromide; PARP: Poly (ADP-ribose) polymerase; PBS: Phosphate-buffered saline; Rh123: Rhodamine 123; ROS: Reactive oxygen species; TUNEL: Terminal deoxynucleotidyl transferase dUTP nick-end labeling; $\Delta \psi \mathrm{m}$ : mitochondrial membrane potential

\section{Funding}

This work was supported by the National Research Foundation of Korea (NRF) grant funded by the Korea Government [MSIP] (No.2015K1A3A1A59069800) and (No. 2017R1D1A3B03027847), and the NRF grant funded by the Korea government [MSIP] (No. 2012R1A5A2A42671316), and and also by the Grant K18102 awarded to Korea Institute of Oriental Medicine (KIOM) from Korea Ministry of Education, Science and Technology (MEST).
\end{abstract}

\section{Availability of data and materials}

The datasets generated and/or analyzed during this study are available from the corresponding author on reasonable request.

\section{Authors' contributions}

E.J.J., S.C.K., J.H.L. and S.Y.B. conducted research and cell experiments of fucoxanthin and L. japonica. J.R.L. analyzed the contents of fucoxanthin in $L$. japonica. S.C.K. and S.Y.B. helped the writing the paper and the analysis of data. E.J.J., J.H.L., S.Y.B., I.K.K. and Y.W.K designed research and wrote the paper. S.C.K., S.Y.B. and Y.W.K. supported financial funding. All authors read and approved the final manuscript.

\section{Ethics approval and consent to participate}

Not applicable

\section{Consent for publication}

Not applicable

\section{Competing interests}

The authors declare that they have no competing interests.

\section{Publisher's Note}

Springer Nature remains neutral with regard to jurisdictional claims in published maps and institutional affiliations.

\section{Author details}

${ }^{1}$ College of Oriental Medicine, Daegu Haany University, Gyeongsan, Gyeongsangbuk-do 38610, South Korea. ${ }^{2}$ College of Korean Medicine, Dongguk University, Gyungju, Gyeongbuk 38066, South Korea. ${ }^{3}$ Kyungpook National University, Daegu 41566, South Korea.

Received: 31 August 2017 Accepted: 8 March 2018

Published online: 20 March 2018

\section{References}

1. Wang J, Zhang Q, Zhang Z, Zhang H, Niu X. Structural studies on a novel fucogalactan sulfate extracted from the brown seaweed Laminaria japonica. Int J Biol Macromol. 2010;47(2):126-31.

2. Kang SI, Shin HS, Kim HM, Yoon SA, Kang SW, Kim JH, Ko HC, Kim SJ. Petalonia binghamiae extract and its constituent fucoxanthin ameliorate high-fat diet-induced obesity by activating AMP-activated protein kinase. J Agric Food Chem. 2012;60:3389-95.

3. Peng J, Yuan JP, Wu CF, Wang JH. Fucoxanthin, a marine carotenoid present in brown seaweeds and diatoms: metabolism and bioactivities relevant to human health. Mar Drugs. 2011;9:1806-28.

4. Rengarajan T, Rajendran P, Nandakumar N, Balasubramanian MP, Nishigaki I. Cancer preventive efficacy of marine carotenoid fucoxanthin: cell cycle arrest and apoptosis. Nutrients. 2013;5:4978-89.

5. Sangeetha RK, Bhaskar N, Baskaran V. Comparative effects of beta-carotene and fucoxanthin on retinol deficiency induced oxidative stress in rats. Mol Cell Biochem. 2009;331:59-67.

6. Liu CL, Liang AL, Hu ML. Protective effects of fucoxanthin against ferric nitrilotriacetate-induced oxidative stress in murine hepatic BNL CL.2 cells. Toxicol Vitro. 2011;25:1314-9.

7. Seo MJ, Seo YJ, Pan CH, Lee OH, Kim KJ, Lee BY. Fucoxanthin suppresses lipid accumulation and ROS production during differentiation in 3T3-L1 adipocytes. Phytother Res. 2016;30:1802-8.

8. Gough DR, Cotter TG. Hydrogen peroxide: a Jekyll and Hyde signalling molecule. Cell Death Dis. 2011;2:e213.

9. Li S, Tan HY, Wang N, Zhang ZJ, Lao L, Wong CW, Feng Y. The role of oxidative stress and antioxidants in liver diseases. Int J Mol Sci. 2015;16:26087-124.

10. Hardie DG, Ross FA, Hawley SA. AMPK: a nutrient and energy sensor that maintains energy homeostasis. Nat Rev Mol Cell Biol. 2012;3:251-62.

11. Sid B, Verrax J, Calderon PB. Role of AMPK activation in oxidative cell damage: implications for alcohol-induced liver disease. Biochem Pharmacol. 2013; 86:200-9.

12. Filomeni G, De Zio D, Cecconi F. Oxidative stress and autophagy: the clash between damage and metabolic needs. Cell Death Differ. 2015;22:377-88.

13. Gwinn DM, Shackelford DB, Egan DF, Mihaylova MM, Mery A, Vasquez DS, Turk BE, Shaw RJ. AMPK phosphorylation of raptor mediates a metabolic checkpoint. Mol Cell. 2008;30:214-26.

14. Kim J, Kundu M, Viollet B, Guan KL. AMPK and mTOR regulate autophagy through direct phosphorylation of UIk1. Nat Cell Biol. 2011;13:132-41.

15. Mizushima N, Levine B, Cuervo AM, Klionsky DJ. Autophagy fights disease through cellular self-digestion. Nature. 2008;451:1069-75.

16. Cui J, Gong Z, Shen HM. The role of autophagy in liver diseases: molecular mechanisms and potential therapeutic targets. Biochim Biophys Acta. 2013; 1836(1):15-26

17. Yeganeh B, Rezaei Moghadam A, Alizadeh J, Wiechec E, Alavian SM, Hashemi M, Geramizadeh B, Samali A, Bagheri Lankarani K, Post M, Peymani P, Coombs KM, Ghavami S. Hepatitis B and C virus-induced hepatitis: apoptosis, autophagy, and unfolded protein response. World I Gastroenterol. 2015;21(47):13225-39.

18. Lee YJ, Jang BK. The role of autophagy in hepatocellular carcinoma. Int J Mol Sci. 2015;16:26629-43.

19. Komatsu M, Waguri S, Ueno T, Iwata J, Murata S, Tanida I, Ezaki J, Mizushima N, Ohsumi Y, Uchiyama Y, Kominami E, Tanaka K, Chiba T. Impairment of starvation-induced and constitutive autophagy in Atg7-deficient mice. J Cell Biol. 2005; 169:425-34.

20. Seo HL, Baek SY, Lee EH, Lee JH, Lee SG, Kim KY, Jang MH, Park MH, Kim JH, Kim KJ, Lee HS, Ahn SC, Lee JR, Park SJ, Kim SC, Kim YW. Liqustri lucidi Fructus inhibits hepatic injury and functions as an antioxidant by activation of AMPactivated protein kinase in vivo and in vitro. Chem Biol Interact. 2017;262:57-68. 
21. Choi SH, Kim YW, Kim SG. AMPK-mediated GSK3beta inhibition by isoliquiritigenin contributes to protecting mitochondria against ironcatalyzed oxidative stress. Biochem Pharmacol. 2010;79(9):1352-62.

22. Anderson ER, Shah YM. Iron homeostasis in the liver. Compr Physiol. 2013;3:315-30.

23. Shin SM, Kim SG. Inhibition of arachidonic acid and iron-induced mitochondrial dysfunction and apoptosis by oltipraz and novel 1,2-dithiole-3-thione congeners. Mol Pharmacol. 2009;75:242-53.

24. Chen Y, Azad MB, Gibson SB. Methods for detecting autophagy and determining autophagy-induced cell death. Can J Physiol Pharmacol. 2010; 88:285-95.

25. Kim YW, Lee SM, Shin SM, Hwang SJ, Brooks JS, Kang HE, Lee MG, Kim SC, Kim SG. Efficacy of sauchinone as a novel AMPK-activating lignan for preventing iron-induced oxidative stress and liver injury. Free Radic Biol Med. 2009;47:1082-92.

26. Shin SM, Cho IJ, Kim SG. Resveratrol protects mitochondria against oxidative stress through AMP-activated protein kinase-mediated glycogen synthase kinase-3beta inhibition downstream of poly(ADP-ribose)polymerase-LKB1 pathway. Mol Pharmacol. 2009;76:884-95.

27. Levine B, Kroemer G. Autophagy in the pathogenesis of disease. Cell. 2008, 132(1):27-42.

28. Kang R, Zeh HJ, Lotze MT, Tang D. The beclin 1 network regulates autophagy and apoptosis. Cell Death Differ. 2011;18:571-80.

29. Das SK, Hashimoto T, Kanazawa K. Growth inhibition of human hepatic carcinoma HepG2 cells by fucoxanthin is associated with down-regulation of cyclin D. Biochim Biophys Acta. 2008;1780:743-9.

\section{Submit your next manuscript to BioMed Central} and we will help you at every step:

- We accept pre-submission inquiries

- Our selector tool helps you to find the most relevant journal

- We provide round the clock customer support

- Convenient online submission

- Thorough peer review

- Inclusion in PubMed and all major indexing services

- Maximum visibility for your research

Submit your manuscript at www.biomedcentral.com/submit 